\title{
Quenching of Star Formation in Molecular Outflow Host NGC 1266
}

K. Alatalo ${ }^{1,16}$, K. E. Nyland ${ }^{2}$, G. Graves ${ }^{1}$, S. Deustua ${ }^{3}$, L. M. Young ${ }^{2}$, T. A. Davis ${ }^{4}$ A. F. Crocker $^{5}$, M. Bureau ${ }^{6}$, E. Bayet ${ }^{6}$ L. Blitz ${ }^{2}$ M. Bois ${ }^{7}$ F. Bournaud, ${ }^{8}$, M. Cappellari,${ }^{6}$ R. L. Davies,${ }^{6}$ P. T. de Zeeuw, ${ }^{4,13}$ E. Emsellem, ${ }^{4,17}$ S. Khochfar, ${ }^{9}$ D. Krajnovic, ${ }^{4}$ H. Kuntschner, ${ }^{4}$ R. M. McDermid,${ }^{10}$ R. Morganti, ${ }^{11}$ T. Naab,${ }^{9}$ T. Oosterloo, ${ }^{11}$ M. Sarzi,${ }^{12}$ N. Scott, ${ }^{14}$ P. Serra ${ }^{12}$ and A. Weijmans ${ }^{15}$

${ }^{1}$ University of California, Berkeley, USA; email: kalatalo@ipac.caltech.edu; ${ }^{2}$ New Mexico Tech, Socorro, USA; ${ }^{3}$ Space Telescope Science Institute, Baltimore, USA; ${ }^{4}$ ESO, Garching, Germany; ${ }^{5}$ University of Toledo, USA; ${ }^{6}$ University of Oxford, UK; ${ }^{7}$ Observatoire de Paris, France; ${ }^{8}$ Université Paris Diderot, France; ${ }^{9} \mathrm{MPI}$ for Extraterrestrial Physics, Garching, Germany; ${ }^{10}$ Gemini Observatory, Hilo, USA; ${ }^{11}$ ASTRON, Dwingeloo, The Netherlands;

${ }^{12}$ University of Hertfordshire, Hatfield, UK; ${ }^{13}$ Leiden University, The Netherlands;

${ }^{14}$ Swinburne University, Australia; ${ }^{15}$ University of Toronto, Canada; ${ }^{16}$ IPAC/Caltech, USA; ${ }^{17}$ Université de Lyon, France

\begin{abstract}
We detail the rich molecular story of NGC 1266, its serendipitous discovery within the ATLAS ${ }^{3 \mathrm{D}}$ survey (Cappellari et al. 2011) and how it plays host to an AGN-driven molecular outflow, potentially quenching all of its star formation (SF) within the next 100 Myr. While major mergers appear to play a role in instigating outflows in other systems, deep imaging of NGC 1266 as well as stellar kinematic observations from SAURON, have failed to provide evidence that NGC 1266 has recently been involved in a major interaction. The molecular gas and the instantaneous SF tracers indicate that the current sites of star formation are located in a hypercompact disk within $200 \mathrm{pc}$ of the nucleus (Fig. 1; SF rate $\approx 2 M_{\odot} \mathrm{yr}^{-1}$ ). On the other hand, tracers of recent star formation, such as the $\mathrm{H} \beta$ absorption map from SAURON and stellar population analysis show that the young stars are distributed throughout a larger area of the galaxy than current star formation. As the AGN at the center of NGC 1266 continues to drive cold gas out of the galaxy, we expect star formation rates to decline as the star formation is ultimately quenched. Thus, NGC 1266 is in the midst of a key portion of its evolution and continued studies of this unique galaxy may help improve our understanding of how galaxies transition from the blue to the red sequence (Alatalo et al. 2011).
\end{abstract}

Keywords. galaxies: evolution — galaxies: individual (NGC 1266) — galaxies: active
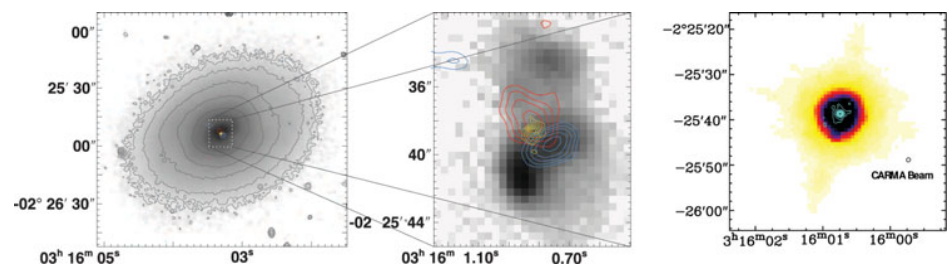

Figure 1. (Left) The molecular outflow of NGC 1266 is shown on an $R$-band image, showing that its detected extent is quite small. The zoomed-in image shows the same molecular gas overlaid on $\mathrm{H} \alpha$. (Right) The central molecular gas is overlaid on the Spitzer $8 \mu \mathrm{m}$ PAH emission.

\section{References}

Alatalo, K., et al. 2011, ApJ, 735, 88

Cappellari, M. et al. 2011, MNRAS, 413, 813 